\title{
Per un metodo d'analisi della pubblicità
}

\author{
Vanni CODELUPPI ${ }^{1}$ \\ Università di Modena e Reggio Emilia
}

Recibido: 04/03/2013

Aceptado: 22/05/2013

\begin{abstract}
Riassunto
La semiotica è la disciplina che ha prodotto la maggior quantità di strumenti utili per comprendere $\mathrm{i}$ significati espressi dai messaggi pubblicitari. Pertanto, pur all'interno di una prospettiva di tipo interdisciplinare, in questo articolo, dopo avere passato in rassegna i contributi storicamente più importanti per quanto riguarda l'analisi semiotica della pubblicità, verrà presentato un modello che deriva dalle ricerche sviluppate negli anni scorsi nell'ambito della semiotica. Tale modello è stato specificamente sviluppato per interpretare i messaggi pubblicitari e in seguito ne verrà mostrata l'efficacia applicandolo al celebre caso Panzani, già analizzato da Roland Barthes negli anni Sessanta.
\end{abstract}

Parole Chiave: Pubblicità, marca, semiotica, linguaggio, comunicazione.

\section{Towards a model for the analysis of advertising}

\begin{abstract}
Semiotics is the discipline that has produced the greater amount of tools to explain the meanings of advertising messages. Therefore, this article adopt an interdisciplinary perspective, but, in particular, presents the most important works about the semiotic analysis of advertising and a new model that descends from the semiotic studies developed in the past years. This model has been specifically created to explain advertising messages and in this article it's also applied to the well-known case of Panzani that Roland Barthes contended in the Sixties.
\end{abstract}

Keywords: advertising, brand, semiotics, language, communication

\section{Introduzione}

Per comprendere i significati espressi dalla comunicazione pubblicitaria, oggi è possibile fare ricorso a numerosi strumenti d'analisi. Strumenti che sono rintracciabili nei contributi elaborati dalle diverse discipline che hanno studiato i linguaggi delle principali forme espressive: psicologia, sociologia, antropologia, semiotica. Quest'ultima però, essendo la disciplina che studia tutti i processi di produzione di senso che si svolgono nella società, è quella che ha fornito la maggior quantità di strumenti utilizzabili per smontare e comprendere i significati che vengono espressi dai messaggi pubblici-

\footnotetext{
${ }^{1}$ Professore di Comunicazione Pubblicitaria all’Università di Modena e Reggio Emilia E-mail: vanni.codeluppi@unimore.it
} 
tari. Anche noi faremo pertanto maggiormente ricorso ad essa, pur all'interno di una prospettiva di tipo interdisciplinare. Anche perché la semiotica possiede sicuramente dei limiti. Per molti anni, ad esempio, ha scelto di adottare una posizione immanente all'oggetto da studiare, cioè il messaggio. Ma all'analisi semiotica della pubblicità sono stati imputati anche altri limiti: l'eccessiva dipendenza dalle capacità interpretative del singolo analista, l'impossibilità di essere applicata con lo stesso successo a tutte le comunicazioni pubblicitarie, l'impiego di un linguaggio e di concetti eccessivamente accademici ed astratti. Ma, con i progressi che ha fatto negli ultimi decenni, la semiotica ha potuto superare una parte consistente di tali limiti. Infatti, gli studi condotti da Algirdas Julien Greimas ${ }^{2}$ e dalla sua scuola teorica - la cosiddetta «Scuola di Parigi» rendono disponibile un insieme sistematico di principi e regole d'analisi. La semiotica continua comunque ad essere una disciplina che è caratterizzata da una condizione di instabilità teorica, anche perché non tutti gli studiosi si sono uniformati al modello della «semiotica generativa» che è stato elaborato dalla scuola greimasiana. Sono almeno altrettanto significativi, ad esempio, i filoni di ricerca della semiotica interpretativa, della semiotica enunciativa e della sociosemiotica. Ma è proprio la mancanza di rigidità teorica della semiotica che fa di questa disciplina uno strumento utile per la ricerca sulla pubblicità, perché la rende maggiormente flessibile e adattabile alle molteplici forme che vengono assunte dai messaggi pubblicitari odierni. D'altronde, nell'analisi dei messaggi pubblicitari non può esistere un approccio teorico più efficace degli altri e da applicare perciò in qualsiasi situazione. Ciascuna forma comunicativa possiede una specifica identità la quale richiede di ricorrere agli strumenti di analisi più adeguati per essa.

Per queste ragioni, nelle pagine che seguono, dopo avere passato in rassegna i contributi storicamente più importanti nell'ambito dell'analisi semiotica della pubblicità, verrà presentato un modello di origine semiotica che è stato specificamente sviluppato per interpretare i messaggi pubblicitari. In seguito, tale modello verrà messo alla prova applicandolo al caso della pubblicità Panzani, resa celebre negli anni Sessanta da Roland Barthes, che ne ha fatto l'oggetto di una sua importante analisi.

\section{La pubblicità e la semiotica}

L'analisi semiotica della pubblicità ha cominciato a svilupparsi negli anni Sessanta, grazie agli sforzi intrapresi da numerosi studiosi per "smontare" i messaggi pubblicitari. A cominciare da Roland Barthes, il quale ha pensato di poter mettere a punto un metodo semiotico utilizzabile per analizzare tutte le forme di comunicazione, che riteneva fossero dotate di strutture narrative comuni. Tra le varie forme comunicative, però, Barthes ha considerato la pubblicità come una delle più importanti, e lo dimostra il ruolo centrale che le ha attribuito sin dal volume Mythologies ${ }^{3}$. Barthes considerava la pubblicità uno strumento in grado di consentire di superare i problemi connessi con l'interpretazione di oggetti statici quali le merci mediante la sua capacità di "mettere in scena", che permette all'analista di isolare la merce rappresentata dalla sua realtà concreta e di analizzare così la merce stessa in uno stato quasi "dinamico" della sua fun-

${ }^{2}$ Si vedano su questo argomento GREIMAS, A.J. (1966); GREIMAS, A.J. (1970); GREIMAS, A.J. (1983).

${ }^{3}$ BARTHES, R. (1957): Mythologies, Paris, Seuil. 
zione comunicativa. Si tratta naturalmente di un'illusione, perché, come ha dimostrato la riflessione successiva, per comprendere come realmente si sviluppano i processi di significazione all'interno del sistema del consumo, è necessario studiare il ruolo attivo giocato dalle merci all'interno del processo di produzione sociale dei significati ${ }^{4}$. Ma Barthes ha attribuito un ruolo fondamentale alla pubblicità anche perché, grazie alla sua natura di comunicazione intenzionale ed esplicita, gli ha permesso di sviluppare il suo personale approccio alla semiotica visiva.

Storicamente, dopo i lavori di Barthes, lo sviluppo in ambito semiotico dell'analisi dei messaggi pubblicitari è proseguito con il volume pubblicato da Georges Péninou nel 1972: Intelligence de la publicité ${ }^{5}$. Tale autore ha individuato la presenza all'interno di un qualsiasi messaggio pubblicitario di cinque principali tipi di sub-messaggi:

- propriamente pubblicitario: è ciò che si percepisce immediatamente nel primo impatto con il messaggio pubblicitario e che permette di identificare la comunicazione pubblicitaria come tale, e dunque permette anche di distinguerla da altre forme di comunicazione;

- dell'emittente: consiste generalmente nel marchio dell'agenzia pubblicitaria, dell'art director o del fotografo e tende generalmente a passare inosservato essendo isolato dal resto della comunicazione pubblicitaria;

- linguistico: presenta al suo interno tre livelli: quello della forma (variabile in base alla natura dei caratteri tipografici: diversità, grandezza, disposizione spaziale, colori, ecc.); quello della lingua, dove, poiché la pubblicità deve sfuggire all'usura comunicativa determinata dalla sua necessità intrinseca di una continua ripetizione quotidiana, le parole vengono sfruttate soprattutto sul piano delle loro capacità semantiche, attraverso varie strategie linguistiche che implicano la creazione di nuovi termini o la trasformazione di slogan, frasi fatte e stereotipi verbali già diffusi socialmente; quello, infine, delle funzioni di ancoraggio o ricambio che, secondo Barthes $^{6}$, le parole svolgerebbero rispetto all'immagine, selezionando e fissando tra i molteplici significati che l'immagine può trasmettere quelli più funzionali ai risultati voluti dall'emittente (ancoraggio) o dicendo ciò che l'immagine non è in grado di esprimere (ricambio);

- iconico: è il messaggio che si percepisce esclusivamente dall'immagine e il suo significato varia in base a come l'immagine stessa occupa lo spazio a disposizione. A seconda infatti della sua distanza rispettivamente dal destinatario o dal prodotto, l'immagine può essere: implicativa (coinvolge il destinatario), predicativa (esalta una qualità della merce), esposizionale (dà conto dell'esistenza di una merce);

- inferenziale: deriva dalla fusione tra messaggio linguistico e messaggio visivo e si realizza soltanto attraverso l'intervento attivo del destinatario. È cioè il piano propriamente connotativo, il più importante per la pubblicità.

Péninou è stato anche uno dei primi autori a tentare un'analisi dinamica delle funzioni svolte dal linguaggio pubblicitario, sulla scia di un analogo tentativo effettuato

\footnotetext{
${ }^{4}$ CODELUPPI, V. (2005), Manuale di Sociologia dei consumi, Roma, Carocci.

${ }^{5}$ PÉNINOU, G. (1976): Semiótica de la publicidad, Barcelona, Gustavo Gili (1972).

${ }^{6}$ BARTHES R. (1964): «Retórica de la imagen », in BARTHES, R., Lo obvio y lo obtuso. Imágenes, gestos, voces, Barcelona, Paidós, 1992 (1982), 30-47.
} 
qualche tempo prima da Umberto $\mathrm{Eco}^{7}$. Ha infatti applicato al linguaggio pubblicitario la classificazione proposta da Roman Jakobson ${ }^{8}$ per le funzioni della comunicazione linguistica. Tali funzioni sono sei e possono essere adattate al discorso pubblicitario come segue:

- referenziale (o denotativa): riguarda il contesto ed è strettamente tesa a denotare merci o servizi fisicamente esistenti;

- emotiva (o espressiva): mira a suscitare delle reazioni emotive nel destinatario attraverso l'inserimento dell'Io dell'emittente nel messaggio pubblicitario;

- conativa (o imperativa): il messaggio rappresenta un comando rivolto direttamente al destinatario, che cerca simultaneamente di rendere attivo quest'ultimo e di coinvolgerlo creando una situazione di complicità;

- fàtica (o contatto): finge di dire o di suscitare emozioni, ma in realtà intende solo verificare lo stabilirsi e il permanere di un canale comunicativo tra l'emittente e il destinatario;

- metalinguistica: riguarda il codice utilizzato per comunicare e consiste in un messaggio che elegge a proprio oggetto un altro messaggio cercando di renderlo intelligibile;

- poetica (o estetica): è relativa al messaggio e si presenta come ambigua perché cerca di attirare l'attenzione del destinatario anzitutto attraverso le innovazioni retoriche formali che la pubblicità introduce rispetto al parlare comune.

Secondo Jakobson, in ogni messaggio possono coesistere tutte e sei le funzioni, o comunque molte di esse, anche se generalmente una soltanto diventa predominante. Eco e Péninou, però, hanno entrambi sostenuto che nel linguaggio pubblicitario è generalmente la funzione emotiva a prevalere, mentre l'importanza delle altre funzioni può essere considerata variabile a seconda del diverso messaggio considerato.

Lamberto Pignotti ed Egidio Mucci ${ }^{9}$ hanno invece sostenuto che in pubblicità le funzioni predominanti sono tre: quella referenziale, quella conativa e quella poetica. Quest'ultima, essendo la pubblicità innanzitutto alla ricerca di un rapporto immediato col destinatario, sembrerebbe non avere una grande importanza a causa della sua ambiguità strutturale, invece è proprio tale sua caratteristica ad attribuirle valore permettendole di disporre di una forte capacità di persuasione. Le rimanenti tre funzioni hanno, sempre secondo Pignotti e Mucci, un'importanza minore in pubblicità: quella emotiva, che è discreta proprio perché basata principalmente sull'Io dell'emittente; quella fàtica, che si confonde spesso con quella poetica e soprattutto con la struttura comunicativa della pubblicità; quella metalinguistica, che può essere considerata addirittura inesistente in questo ambito. La funzione metalinguistica però sta diventando oggi una delle più importanti all'interno della pubblicità, addirittura trasformandola in una vera e propria «metapubblicità» ${ }^{10}$.

${ }^{7}$ ECO, U. (1975): La estructura ausente. Introducción a la semiótica, Barcelona, Lumen (1968).

${ }^{8}$ JAKOBSON, R. (1976), Ensayos de lingüistica general, Barcelona, Seix Barral (1963).

${ }^{9}$ PIGNOTTI, L.; MUCCI E. (1978): Marchio \& Femmina. La donna inventata dalla pubblicità, Firenze, Vallecchi.

${ }^{10} \mathrm{Si}$ vedano su questo argomento CODELUPPI, V. (ed.) (1995); CODELUPPI, V. (2001a). 
Si può dunque essere d'accordo con Roberto Grandi ${ }^{11}$ quando sostiene, dopo aver condotto un'ampia ricerca sulla pubblicità televisiva italiana, che non esiste in realtà una funzione che sia in grado di prevalere sulle altre, soprattutto se si tiene conto dell'intreccio che si è creato nel linguaggio pubblicitario contemporaneo tra gli elementi visivi e quelli sonori, dove tutte le sei funzioni attribuite da Jakobson alla comunicazione linguistica sembrano operare insieme e assumere sostanzialmente lo stesso livello d'importanza.

Ma per comprendere sino in fondo la complessità di funzionamento dei messaggi pubblicitari è necessario ricorrere anche a quegli strumenti teorici e pratici che sono stati sviluppati negli ultimi anni da parte della semiotica, la quale oggi «cerca di ricostruire le logiche interne all'intera comunicazione pubblicitaria, analizzandola in tutte le sue componenti» ${ }^{12}$. Nelle pagine che seguono cercheremo di spiegare come per la semiotica di oggi sia possibile raggiungere questo obiettivo.

\section{Un modello per l'analisi della pubblicità}

Per analizzare l'azione della comunicazione pubblicitaria, abbiamo messo a punto uno specifico modello interpretativo (cfr. Figura 1). Tale modello distingue innanzitutto un'analisi statica, che ha come oggetto la forma e il contenuto dell'enunciato o messaggio, e un'analisi dinamica, che si concentra invece sui processi enunciativi (o enunciazione) riguardanti il funzionamento dell'enunciato stesso, il quale tenta costantemente di stabilire un legame con il destinatario al quale si rivolge. Tali due tipi di analisi fanno riferimento a quelle che sono state riconosciute da tempo come le dimensioni fondamentali di qualsiasi linguaggio. Ovvero la dimensione sintattica (o forma) e semantica (o contenuto) per l'analisi dell'enunciato e la dimensione pragmatica per l'analisi dell'enunciazione.

Il modello individua in seguito due livelli (delle manifestazioni superficiali e narrativo) per la componente sintattica dell'enunciato e due livelli (tematico e valoriale) per la componente semantica dell'enunciato. Individua inoltre sia per la componente sintattica che per quella semantica e quella pragmatica degli specifici strumenti d'analisi che ora andremo dettagliatamente ad illustrare: il modello attanziale, il quadrato semiotico e il modello comunicativo della marca.

${ }^{11}$ GRANDI, R. (1987): Come parla la pubblicità. Modelli comunicativi degli spot pubblicitari, Milano, Edizioni del Sole 24 Ore.

${ }^{12}$ MARRONE, G. (2001): Corpi sociali. Processi comunicativi e semiotica del testo, Torino, Einaudi, 160. 
Fig. 1. Un modello per l'analisi della pubblicità

Analisi statica dell'enunciato (sintattica e semantica)

\begin{tabular}{|c|c|c|c|}
\hline & $\begin{array}{c}\text { Livelli } \\
\text { dell'enunciato }\end{array}$ & Caratteristiche & Strumenti di analisi \\
\hline $\begin{array}{c}\text { Componente } \\
\text { sintattica } \\
\text { (forma) }\end{array}$ & $\begin{array}{c}\text { 1. Livello delle mani- } \\
\text { festazioni superficiali }\end{array}$ & $\begin{array}{c}\text { Codici materiali (co- } \\
\text { lori, impaginazione, } \\
\text { gestualità, prosse- } \\
\text { mica, sonoro, ecc.), } \\
\text { figure retoriche, } \\
\text { modalità stilistiche, } \\
\text { modalità argomen- } \\
\text { tative }\end{array}$ & \\
\hline $\begin{array}{c}\text { Componente } \\
\text { semantica } \\
\text { (contenuto) }\end{array}$ & 2. Livello narrativo & Struttura narrativa & Modello attanziale \\
\hline & 2. Livello tematico & $\begin{array}{c}\text { Denotazioni, conno- } \\
\text { tazioni, temi essen- } \\
\text { ziali, figure }\end{array}$ & \\
\hline
\end{tabular}

Analisi dinamica dell'enunciazione (pragmatica)

\begin{tabular}{|l|l|l|}
\hline & Caratteristiche & Strumenti di analisi \\
\hline $\begin{array}{l}\text { Componente pragmatica } \\
\text { (azione) }\end{array}$ & $\begin{array}{l}\text { Enunciatore, enunciatario, } \\
\text { relazione tra enunciatore e } \\
\text { enunciatario }\end{array}$ & $\begin{array}{l}\text { Modello comunicativo della } \\
\text { marca }\end{array}$ \\
\hline
\end{tabular}

\subsection{Il modello attanziale}

La semiotica ha sviluppato, soprattutto nel corso degli anni Sessanta e Settanta, un'ampia esperienza di analisi rispetto a quelle manifestazioni superficiali che rappresentano il primo livello della componente sintattica del messaggio. Ma per l'analisi di tale componente è utile concentrarsi sul suo secondo livello: quello narrativo. Livello che può essere interpretato facendo ricorso allo schema che Algidas Julien Greimas ha sviluppato a partire dalle categorie individuate in precedenza da Vladimir Propp ${ }^{13}$ all'interno delle fiabe popolari russe. In particolare, Greimas ha messo a punto uno schema interpretativo applicabile a tutte le forme di narrazione e denominato «modello attanziale» (cfr. Figura 2). Si tratta di uno schema basato sulle relazioni di opposizione esistenti tra sei fond amentali tipi di attanti o ruoli narrativi. Greimas ha operato infatti, all'interno della narrazione, una distinzione tra gli attanti, che operano su un piano narrativo astratto, e gli attori, cioè coloro che vivono la storia sul piano empirico.

${ }^{13}$ PROPP, V.J. (1974): Morfología del cuento fiaba, Madrid, Fundamentos (1928). 
Fig. 2. Il modello attanziale

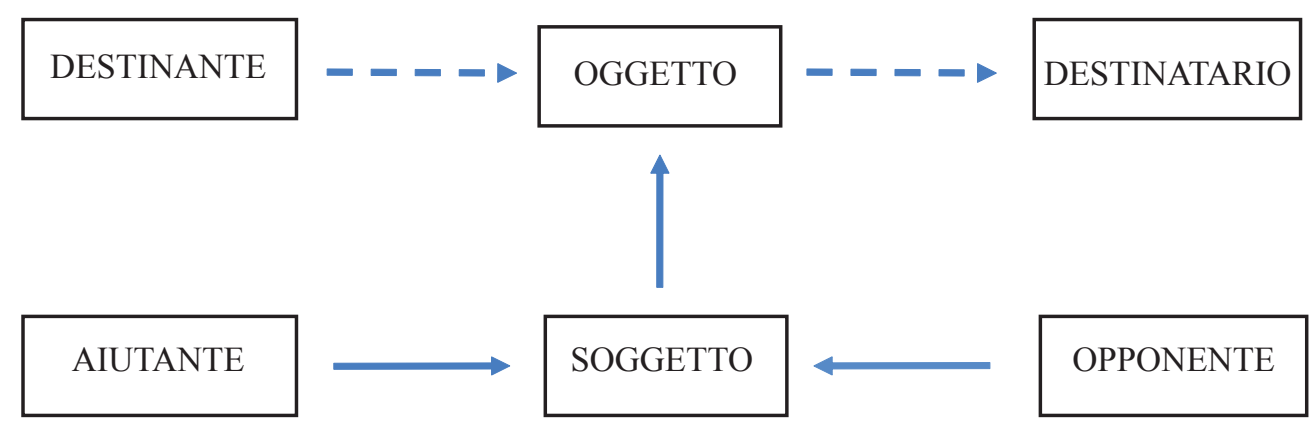

Nel modello attanziale, gli attanti sono accoppiati a due a due e si tratta in particolare di:

- un Soggetto, che cerca di compiere un'impresa superando delle difficoltà per congiungersi con un oggetto nel quale è investito un valore che lo determina, cioè per congiungersi con un Oggetto di valore;

- un Opponente, che si oppone al programma del Soggetto, il quale è assistito da un Aiutante;

- un Destinante, che rappresenta solitamente il mandante del Soggetto all'inizio della narrazione, e il Destinatario, a cui viene affidato alla fine l'Oggetto.

Gli studiosi danesi Torben Vestergaard e Kim Schrøder hanno sostenuto, a tale proposito, che nei messaggi pubblicitari, a differenza di quello che potrebbe apparentemente sembrare, «the role of Object is not filled by the product but by some quality or state associate with it, and that the consumer (the 'you') is both subject and reeciver» ${ }^{14}$. Questo perché la pubblicità non cerca generalmente di convincere il consumatore che ha bisogno dei prodotti pubblicizzati in quanto tali, ma piuttosto che questi prodotti possono aiutarlo ad ottenere qualcos'altro di cui sente di avere la necessità, come ad esempio la salute. L'argentino Juan Magariños de Morentin ${ }^{15}$ ha invece diversamente sostenuto che nei testi pubblicitari non esistono ruoli narrativi fissi e che il prodotto, ad esempio, può occupare uno qualsiasi dei sei ruoli che sono previsti dal modello attanziale di Greimas. Un'opinione che è condivisa anche da Ugo Volli ${ }^{16}$.

Secondo Greimas, inoltre, l'Oggetto di valore è generalmente desiderato, allo stesso tempo, da più Soggetti, che entrano pertanto in una relazione di natura polemico-conflittuale, perché ognuno di essi è mosso da differenti e spesso contrastanti programmi narrativi. L'introduzione del concetto di programma narrativo consente di mettere in luce le diverse possibilità prospettiche della narrazione, che può così essere raccontata

${ }^{14}$ VESTERGAARD, T.; SCHRØDER, K. (1985): The Language of Advertising, Oxford, Basil Blackwell, 29.

${ }^{15}$ MAGARIÑOS DE MORENTIN, J.A. (1984): El mensaje publicitario, Buenos Aires, Hachette.

${ }^{16}$ VOLLI, U. (2003): Semiotica della pubblicità, Roma-Bari, Laterza. 
a partire sia dal programma d'azione del Soggetto, che da quello dell'anti-soggetto, cioè secondo il rispettivo punto di vista di ciascuno dei principali protagonisti del messaggio. Ma è importante soprattutto osservare che il desiderio dell'Oggetto di valore instaura la circolazione di quest'ultimo all'interno del modello attanziale, ovvero una serie di congiunzioni e disgiunzioni successive dell'Oggetto con il Soggetto e i suoi diversi anti-soggetti.

Greimas ha utilizzato i risultati delle ricerche condotte da Propp anche per mettere a punto uno schema del percorso narrativo del Soggetto che rappresenta una semplificazione del modello attanziale visto precedentemente, in quanto non considera i ruoli dell'Opponente e dell'Aiutante. Tale schema prevede quattro successive fasi, ovvero una manipolazione e tre specifiche prove, le quali, considerate isolatamente, costituiscono il cosiddetto «schema narrativo canonico»:

- la manipolazione (che spesso assume la forma di un contratto): proposta da parte del Destinante e accettazione da parte del Soggetto di un programma che dev'essere portato a termine e che prevede delle azioni da compiere e uno o più oggetti da raggiungere e possedere (Oggetti di valore); può essere di tipo unilaterale, bilaterale o reciproco;

- la competenza: acquisizione e riconoscimento della capacità cognitiva e pratica di realizzare il programma da parte del Soggetto (o prova qualificante);

- la performanza: realizzazione del programma proposto (o prova decisiva), che ha generalmente come scopo la congiunzione del Soggetto con l'Oggetto di valore;

- la sanzione: nuovo confronto con il Destinante (questa volta nel ruolo di Destinatario) per la verifica del programma che è stato realizzato rispetto a ciò che era previsto dal contratto (o prova glorificante), allo scopo di emettere un giudizio sull'operato del Soggetto, che può essere di natura positiva (premio o riconoscimento) o di natura negativa (punizione o disconoscimento).

Volli ha sostenuto che «ogni momento di questa sequenza può fallire e concludere la storia, o richiedere ripetizioni, e che spesso lo schema si ripete ricorsivamente, annidato in se stesso» ${ }^{17}$. Pertanto, in pubblicità accade frequentemente che la necessità di disporre di messaggi brevi e convincenti imponga di non fare ricorso ad alcune fasi previste all'interno dello schema narrativo canonico. Ma ciò che soprattutto conta in pubblicità è che il livello narrativo tende a svolgere la funzione di persuadere lo spettatore a comportarsi come il protagonista del messaggio, a seguire il percorso che porta verso il valore promesso.

\subsection{Il quadrato semiotico}

Passando all'analisi dei contenuti, ovvero della componente semantica dell'enunciato, emerge innanzitutto la capacità di esprimere diversi temi. Bruno Remaury ${ }^{18}$ ha individuato a tale proposito sei differenti aree tematiche che i racconti relativi alle marche possono sviluppare:

\footnotetext{
${ }^{17}$ Ibidem, 48-49.
}

${ }^{18}$ REMAURY, B. (2004): Marques et récits. La marque face à l'imaginaire culturel contemporain, Paris, Institut Français de la Mode-Regard. 
- il tempo, cioè racconti che recuperano l'aspetto originario che qualifica la marca;

- i luoghi, cioè racconti fortemente connessi con una dimensione geografica ben determinata;

- gli stati esistenziali, come per esempio l'adolescenza e la femminilità;

- i personaggi, come ad esempio il cowboy della Marlboro;

- il saper-fare, come il mondo di marca di Nike, o i mestieri qualificanti, come l'artigiano di Hermés;

- la materia, da quella naturale passando per quella tecnologica, fino ai componenti brevettati dalla cosmetica.

Per analizzare la componente semantica dell'enunciato è però utile soprattutto fare ricorso a uno schema di tipo logico che consente di raffigurare dinamicamente le principali tra le possibili articolazioni di una qualunque categoria semantica. Tale schema ha delle origini che possono essere ricondotte al pensiero filosofico di Aristotele ed è noto come «quadrato semiotico» (cfr. Figura 3). I quattro poli di tale quadrato sono collegati tra loro da relazioni di natura differente: contrarietà (sui lati orizzontali), complementarietà (sui lati verticali) e contraddizione (sulle diagonali).

Fig. 3. Il quadrato semiotico

S1

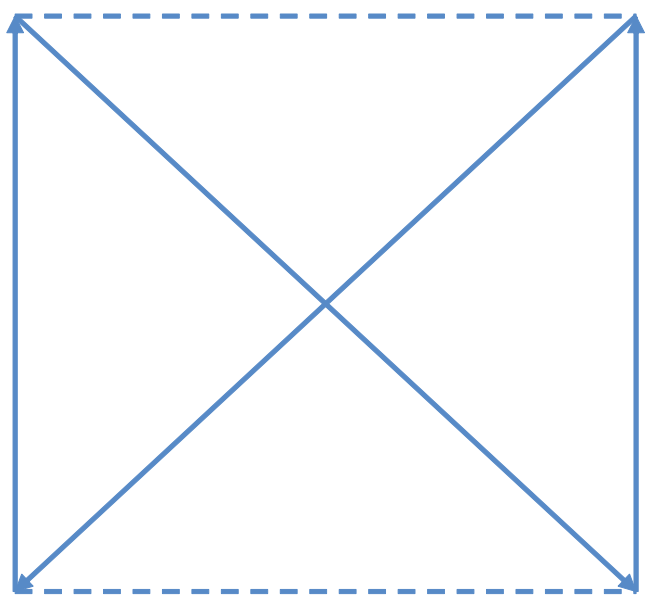

non-S2

non-S1

L'importanza del quadrato semiotico risiede anche nel fatto che tale strumento permette di articolare quei concetti contrapposti che secondo gli antropologi strutturalisti rappresentano i nuclei fondativi della cultura sociale (vita e morte, amore e odio, maschile e femminile, ecc.). Proprio per questo motivo, è qui che risiede il vero nucleo di base del posizionamento pubblicitario di un prodotto o una marca. Posizionare significa 
infatti definire uno spazio concettuale nel quale vanno a rapportarsi tra loro degli elementi come i segni, i simboli, i ricordi e le esperienze che sono relativi a un prodotto o una marca, ovvero creare una determinata posizione nella mente di chi riceve il messaggio, ma significa soprattutto ancorare tali elementi a un valore che dev'essere unico e distintivo per ciascun prodotto/marca e deve anche essere sufficientemente universale da poter venire compreso e apprezzato da parte di ampie fasce di consumatori, o perlomeno da parte di coloro che fanno parte di quello specifico pubblico che si vuole raggiungere.

Jean-Marie Floch ${ }^{19}$, che è stato un importante collaboratore di Greimas, ha impiegato il quadrato semiotico per analizzare numerosi fenomeni di consumo. Floch, in particolare, ha individuato, e verificato sperimentalmente mediante apposite ricerche condotte su campioni di consumatori, quattro tipi di possibili «valorizzazioni dei beni di consumo». Tali valorizzazioni, che si trovano sui diversi angoli del quadrato semiotico (cfr. Figura 4), sono le seguenti:

- pratica, relativa al valore d'uso e all'utilità del bene: confort, affidabilità, ecc.;

- utopica, relativa agli aspetti esistenziali del rapporto di consumo: identità, avventura, ecc. (come sottolinea lo stesso Floch, qui utopico non è da intendersi con il significato di illusorio, ma nel senso di tensione ideale);

- critica, relativa agli aspetti non-esistenziali del consumo: rapporto qualità-prezzo, rapporto costo-benefici, esame circostanziato del bene, ecc;

- ludica, relativa agli aspetti non utilitari: gratuità, raffinatezza, ecc. (qui Floch con l'aggettivo «ludico» vuole intendere l'attività libera per eccellenza, non semplicemente quella che ha a che fare con il gioco).

Fig. 4. Le valorizzazioni dei beni di consumo

PRATICA

valori utilitari

CRITICA

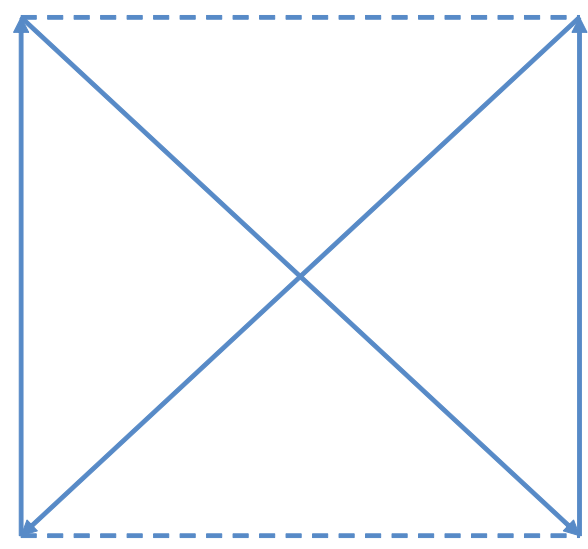

valori non-esistenziali
UTOPICA

valori esistenziali

\section{LUDICA}

valori non-utilitari

${ }^{19}$ FLOCH, J.-M. (1993), Semiótica, marketing y comunicación. Bajo los signos, las estrategias, Barcelona, Paidós (1991). 
Fig. 5. Le ideologie pubblicitarie

REFERENZIALE

funzione rappresen-

tazionale del

linguaggio

SOSTANZIALE

funzione costruttiva denegata
MITICA

funzione costruttiva del linguaggio

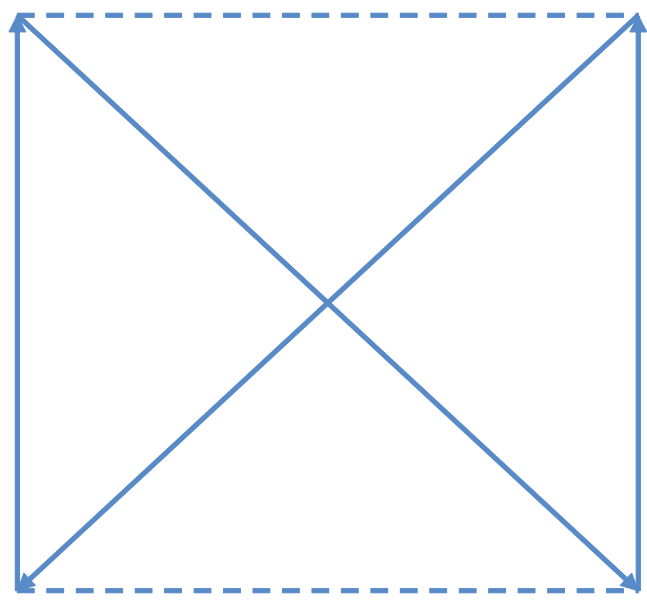

funzione rappresentazionale denegata

Queste quattro valorizzazioni comportano, evidentemente, altrettanti modelli di consumo, ma è importante ricordare in questa sede soprattutto l'impiego del quadrato semiotico che è stato fatto da Floch in relazione ai messaggi pubblicitari, i quali sono in grado solitamente di associare dei valori a prodotti acquistabili sul mercato per renderli desiderabili. Tale impiego ha consentito infatti d'individuare quattro diverse ideologie pubblicitarie (cfr. Figura 5):

- referenziale: il linguaggio pubblicitario vi svolge una funzione rappresentativa, ovvero di semplice rappresentazione di una realtà che è già dotata di un significato (quello del prodotto). I seguaci di questo tipo di filosofia pubblicitaria, a cominciare da David Ogilvy, sostengono che la pubblicità deve limitarsi a rispecchiare la realtà del prodotto (spesso attraverso il meccanismo della dimostrazione). Floch ha giustamente sottolineato però che la pubblicità referenziale appare come tale perché utilizza determinate strategie discorsive che hanno l'obiettivo di presentarla come vera;

- mitica: il linguaggio vi svolge una funzione costruttiva in quanto il significato non è già presente nella realtà del prodotto, ma viene costruito esclusivamente attraverso il discorso pubblicitario. Ricorre spesso a leggende, eroi, simboli, ovvero a referenti mitici che sono già conosciuti e strutturati e li associa con il prodotto. Il pubblicitario francese Jacques Séguéla, con la sua spettacolare pubblicità, ne è senz'altro il più significativo rappresentante, ma insieme ad esso va citato anche Leo Burnett, che ha creato la celebre campagna Marlboro basata sulla figura del cowboy; 
- sostanziale: è la negazione della pubblicità mitica, considerata in questo caso una forma di pubblicità che usa il prodotto in maniera pretestuosa. Essa si batte invece per far vivere il prodotto in pubblicità, attribuendogli, con le sue virtù, una chiara centralità. L'atto creativo è concepito come una specie di "depurazione" che consente di andare ad esplorare e mettere in luce la natura profonda del prodotto. L'obiettivo è comunque di ottenere un'emozione di tipo estetico. Questa filosofia pubblicitaria ha degli illustri antecedenti in molti storici annunci creati da Rosser Reeves;

- obliqua: è la negazione della pubblicità referenziale, in quanto sostiene che nel messaggio il significato non è già dato e utilizza la forza dell'ironia e del paradosso per attivare la capacità cognitiva del fruitore e stimolare quest'ultimo a co-produrre il significato attraverso una strategia di spostamento, di messa in distanza rispetto al discorso che riguarda le finalità del prodotto. Possiamo trovare un pioniere nell'utilizzo della pubblicità obliqua in Bill Bernbach e in particolare nei numerosi annunci che ha creato negli anni sessanta per il Maggiolino Volkswagen. In generale, comunque, la filosofia della pubblicità obliqua comporta il ricorso a un tipo di pubblicità che attualmente si va sempre più diffondendo e che può essere definita «metapubblicità» ${ }^{20}$.

Va ritenuto però che per Floch «tale modello non andasse inteso come uno schema statico che illustra una serie di possibilità in alternativa fra loro, ma come uno strumento operativo utile a ricostruire i movimenti interni ai testi, le trasformazioni semantiche che sono presenti nelle varie storie pubblicitarie ${ }^{21}$. Infatti, possono essere impiegate tutt'e quattro le ideologie pubblicitarie individuate, anche se di queste solitamente una soltanto è la prevalente e definisce pertanto ciascuna marca come referenziale, mitica, sostanziale oppure obliqua. È evidente d'altronde che di fronte a consumatori come quelli contemporanei, che sono caratterizzati in maniera crescente da comportamenti eclettici e instabili, un modello di questo tipo, ma anche qualsiasi altro modello formalizzato, può incontrare delle difficoltà di applicazione.

I semiotici tendono ad attribuire un carattere di universalità al quadrato semiotico, mentre quest'ultimo non è che uno dei tanti strumenti di questo tipo. Altri potrebbero essere rintracciati. Robert Blanché2 ${ }^{2}$, ad esempio, ha provato a costruire un modello semiotico più complesso del quadrato e basato sulla figura dell'esagono. Tale modello, anziché adottare un sistema di opposizioni binarie, si basa su opposizioni ternarie comprendenti anche un terzo termine medio o neutro. Eric Landowski ${ }^{23}$ ha invece proposto di considerare i poli estremi del quadrato come punti qualsiasi di un unico flusso di significato.

Il quadrato semiotico sembra comunque essere uno strumento particolarmente utile per mettere a fuoco i concetti sui quali si basano gli immaginari delle marche. Può

${ }^{20} \mathrm{Si}$ vedano su questo argomento CODELUPPI, V. (ed.) (1995); CODELUPPI, V. (2001a).

${ }^{21}$ MARRONE, G. (2001): Corpi sociali. Processi comunicativi e semiotica del testo, Torino, Einaudi, 182.

${ }^{22}$ BLANCHÉ, R. (1966): Structures intellectuelles. Essai sur l'organisation systématique des concepts, Paris, Vrin.

${ }^{23}$ LANDOWSKI, E. (2006): «Les Interactions risquées», Nouveaux Actes Sémiotiques, 101-103. 
dunque essere efficacemente utilizzato, naturalmente tenendo presente che, come tutti i modelli scientifici, è una rappresentazione visiva di tipo convenzionale.

\subsection{Il modello comunicativo della marca}

Per comprendere come operano sul piano comunicativo i fenomeni di consumo, è stato da tempo proposto un apposito modello interpretativo. Tale modello, che ha avuto negli anni diverse versioni ${ }^{24}$, innesta il contributo interpretativo proprio della sociosemiotica all'interno della tradizione proveniente dalla teoria dell'informazione e dagli studi sulla comunicazione. È stato messo a punto per consentire di analizzare tutti i fenomeni comunicativi che possono presentarsi nel mondo del consumo e può dunque essere impiegato per comprendere il funzionamento della pubblicità e della marca (cfr. Figura 6).

Fig. 6. Il modello comunicativo della marca

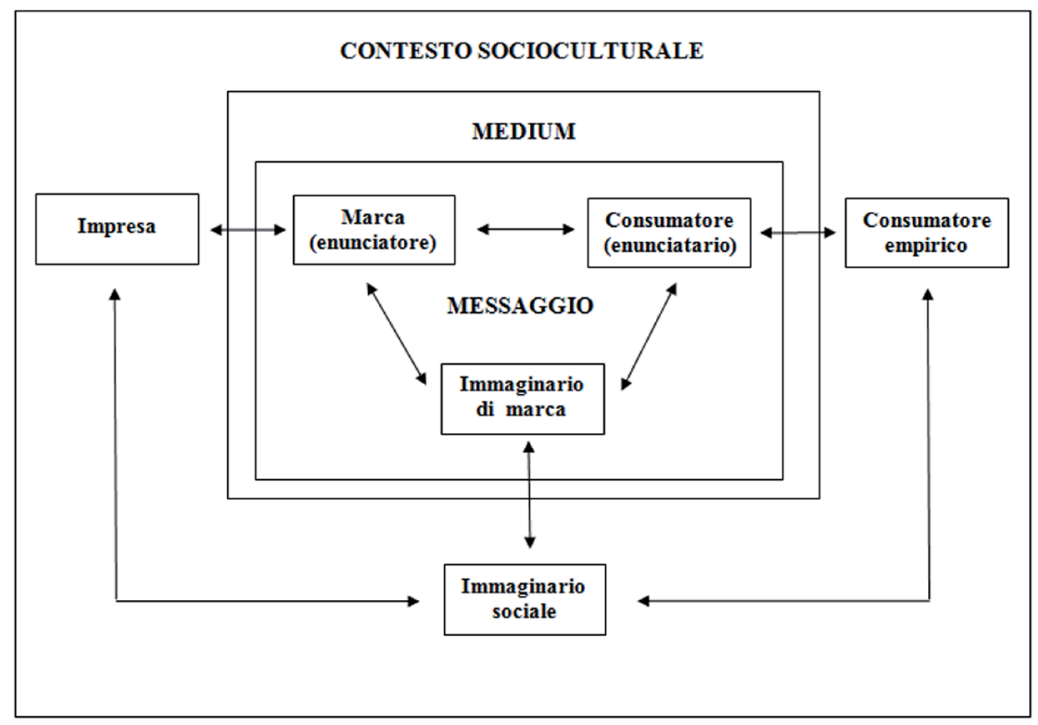

Vale la pena di presentare le principali caratteristiche di tale modello, che si ispira a quello sviluppato da Roman Jakobson ${ }^{25}$ per la comunicazione linguistica, modello che deriva a sua volta da quello messo a punto precedentemente da Shannon e Weaver ${ }^{26}$. A differenza però di tali modelli, il tipo di rappresentazione adottato non è lineare, in quanto utilizza una grafica di tipo "insiemistico" allo scopo di sottolineare la forte interattività esistente tra tutti i soggetti attivi al suo interno. Come mostra la figura, similmente a qualsiasi altro modello relativo alla comunicazione, abbiamo innanzitutto un emittente empirico (l'impresa) e un destinatario empirico (il consumatore). Si tratta di soggetti che fanno sempre riferimento a un determinato contesto socioculturale e sono

${ }^{24}$ CODELUPPI, V. (1989); CODELUPPI, V. (1997); CODELUPPI, V. (2005).

${ }^{25}$ JAKOBSON, R. (1976), Ensayos de lingüistica general, Barcelona, Seix Barral (1963).

${ }^{26}$ SHANNON, C.E.; WEAVER, W. (1949): The Mathematical Theory of Communication, Urbana, University of Illinois Press. 
collegati tra di loro da un medium che consente la circolazione di messaggi.

La figura mostra anche che i messaggi comprendono al loro interno, come ogni altra forma di messaggio, due ruoli astratti che rappresentano simbolicamente l'emittente e il destinatario empirici: l'enunciatore (la marca) e l'enunciatario (il consumatore rappresentato). Come ha spiegato infatti Gianfranco Bettetini,

- l'enunciatore è l'immagine di sé che viene prodotta dall'emittente empirico ed è il soggetto più importante in quanto, essendo «produttore e prodotto del testo, è l'origine del discorso che vi si svolge, la fonte del sapere che vi si trasmette e lo stratega dei percorsi di senso che vi si attuano ${ }^{27}$;

- l'enunciatario è invece l'immagine del recettore che il testo si costruisce.

L'enunciatore, l'enunciatario e la relazione che li unisce di solito non sono osservabili direttamente, ma soltanto attraverso una serie di tracce di riconoscimento (o marche enunciative) contenute all'interno del messaggio in conseguenza del presentarsi di quel processo di enunciazione che permette al messaggio stesso di esistere. Attraverso l'individuazione e l'analisi di tali tracce è pertanto possibile riconoscere la natura specifica sia dell'enunciatore che dell'enunciatario, ma anche la relazione tra essi che è comportata dal particolare punto di vista adottato dal messaggio (che può valorizzare l'uno, l'altro o nessuno dei due in particolare) e il tipo di rapporto con il messaggio che è previsto per quel destinatario empirico che è chiamato a identificarsi con l'enunciatario.

Le tracce contenute nel messaggio sono rappresentate, nel caso del linguaggio verbale, dai cosiddetti «deittici» (tempi dei verbi, avverbi di luogo e di tempo, pronomi dimostrativi, ecc.), ma anche dagli elementi «sovrasegmentali» (cioè non riducibili ai frammenti nei quali il testo può essere frazionato) ${ }^{28}$. Nel caso invece del linguaggio visivo, le tracce sono costituite da elementi come la composizione figurativa, il gioco degli sguardi dei protagonisti, le angolazioni delle inquadrature, i movimenti di camera, ecc. ${ }^{29}$.

Bettetini $^{30}$ ha anche individuato la presenza all'interno di ogni messaggio di un «enunciatore modello», cioè di quell'immagine ideale dell'emittente che il destinatario si costruisce nel corso del suo rapporto con tale messaggio. Si tratterebbe, secondo Bettetini, di un'immagine attiva soltanto all'interno di un messaggio "chiuso", unidirezionale e che non prevede altri spazi per il destinatario come quello audiovisivo. È invece evidente che un enunciatore modello è presente anche all'interno del nostro modello. Non è stato rappresentato nella figura per non complicarla eccessivamente, anche se va comunque tenuto presente insieme al fatto che potrebbe essere affiancato da un «enunciatario modello», cioè da quell'immagine ideale di sé che il destinatario

${ }^{27}$ BETTETINI, G. (1985): L'occhio in vendita. Per una logica e un'etica della comunicazione audiovisiva, Venezia, Marsilio, 44. 18.

${ }^{28}$ BETTETINI, G. (1993): Semiotica della comunicazione d'impresa, Milano, Bompiani,

${ }^{29} \mathrm{Si}$ vedano su questo argomento CASETTI, F. (1986); CORNU, G. (1991); EUGENI, R. (1999).

${ }^{30}$ BETTETINI, G. (1984): La conversazione audiovisiva. Problemi dell'enunciazione filmica e televisiva, Milano, Bompiani. 
vuole offrire agli altri.

Va considerato inoltre che, all'interno del messaggio, enunciatore ed enunciatario stabiliscono una diretta relazione con un preciso «immaginario di marca» ${ }^{31}$, cioè un universo comunicativo che riproduce e sviluppa una frazione dell'immaginario sociale. Tali due mondi immaginari intrattengono dunque tra loro una dialettica di complementarietà.

In ogni sistema comunicativo, è fondamentale il lavoro svolto dai destinatari empirici presenti, che in questo caso sono rappresentati nella maggior parte dei casi da consumatori. L'emittente opera infatti cercando di inviare dei messaggi al destinatario, ma questi non decodifica passivamente i significati apparentemente contenuti in essi. Al contrario, il destinatario riveste un ruolo estremamente importante nel processo di comunicazione, perché contribuisce in prima persona alla produzione dei significati. Tali significati non sono però da considerare come totalmente soggettivi, perché tra destinatario e messaggi si stabilisce sempre un rapporto di «cooperazione interpretativa» ${ }^{32}$. Il destinatario si trova perciò in una situazione che è, allo stesso tempo, di debolezza, in quanto il suo percorso interpretativo è già previsto all'interno del messaggio, ma anche di forza, perché senza la sua presenza attiva tale percorso rimarrebbe allo stato virtuale e non potrebbe concretizzarsi. È perciò soprattutto grazie ai destinatari che i significati riescono a svilupparsi e circolare nel modello.

Vediamo ora, più specificamente, come operano i principali elementi costitutivi del modello comunicativo della marca, partendo dai due ruoli centrali: l'impresa e il consumatore. Entrambi tali soggetti possono operare sia come emittenti che come destinatari, anche se nella nostra figura abbiamo rappresentato per ragioni di semplicità la situazione più classica: l'impresa come emittente e il consumatore come destinatario. In realtà, la stessa distinzione tra produttore e consumatore tende a perdere sempre più di significato, perché proprio il fatto che la crescita degli aspetti comunicativi del consumo trasformi il tradizionale consumo fisico dei prodotti in un vero e proprio "consumo di significato" accresce la natura produttiva dei consumatori. Infatti, il significato, a differenza di quanto avviene con le componenti fisiche dei prodotti, si produce nel momento stesso del suo consumo, nel corso cioè di quel processo di tipo interattivo che avviene tra il destinatario e il messaggio della marca. A ciò contribuisce naturalmente anche l'evoluzione del sistema economico occidentale, nel quale si sta producendo una crescente interazione tra la produzione e il consumo.

Jakobson ha parlato del contesto considerandolo come il «referente» del testo, ma è necessario oggi vedere il contesto come quella situazione empirica nella quale l'emittente e il destinatario si trovano ad operare e che influenza naturalmente il loro processo comunicativo. Esso è comunque, proprio a causa della sua natura empirica, qualche cosa di esterno al vero sistema comunicativo, una sorta di pre-requisito indispensabile all'attività di tale sistema. Nel sistema comunicativo della marca il contesto è perciò rappresentato dal mercato e dalla società.

In tale sistema comunicativo, il ruolo di medium viene ricoperto dai diversi mezzi di comunicazione impiegati. Mezzi che non si limitano a svolgere un ruolo puramente

${ }^{31}$ CODELUPPI, V. (2001b): Il potere della marca, Torino, Bollati Boringhieri.

${ }^{32}$ ECO, U. (1979): Lector in fabula, Milano, Bompiani. 
strumentale, in quanto sono dotati di una specifica identità che interagisce con il processo di trasmissione ed interpretazione dei messaggi.

I media hanno inoltre il compito di contribuire alla produzione di un altro elemento fondamentale del contesto socioculturale del sistema comunicativo della marca: l'enciclopedia semantica ${ }^{33}$. Si tratta di quel complesso sistema di conoscenze che rende possibile l'attivarsi di un processo comunicativo tra l'emittente e il destinatario, perché comprende e organizza al suo interno numerosi elementi: significati culturali, interpretazioni, definizioni, istruzioni, ecc. Il concetto di enciclopedia prevede, inoltre, anche la presenza di sceneggiature oframes, cioè di modelli prefissati di comportamento legati a situazioni stereotipate e pronti ad attualizzarsi quando la situazione lo richieda.

I messaggi comunque, per poter essere compresi dal destinatario, devono essere costruiti utilizzando almeno una porzione dell'enciclopedia che è posseduta dal destinatario stesso. Infatti, come ha spiegato $\mathrm{Eco}^{34}$, è possibile pensare al concetto astratto di enciclopedia semantica globale soltanto dal punto di vista di una teoria semiotica generale, ma sul concreto piano sociosemiotico è necessario identificare le specifiche «enciclopedie locali» (di gruppo sociale, di classe, di gruppo etnico, ecc.) appartenenti ad ogni soggetto in azione. Dal punto di vista dell'impresa, l'enciclopedia locale rappresenta quell'insieme particolare di miti e conoscenze che appartiene alla cultura dei consumatori di una determinata marca. È da questo specifico tipo di enciclopedia che $\mathrm{i}$ pubblicitari e gli uomini di marketing devono estrarre i materiali culturali attraverso i quali possono costruire un'immagine e un'identità che consenta ad ogni messaggio di raggiungere un'elevata sintonia comunicativa con i consumatori appartenenti al segmento di mercato prescelto.

Tuttavia, le funzioni dell'enciclopedia sono spesso ostacolate da quella strutturale mancanza di simmetria che esiste tra l'enciclopedia propria dell'emittente e quella propria del destinatario, ma, soprattutto, tra l'enciclopedia realmente posseduta dal destinatario e l'enciclopedia immaginata per quest'ultimo dall'emittente quando costruisce il suo enunciatario all'interno del messaggio. Questi problemi di simmetria disturbano infatti il processo di comunicazione e arrivano a distorcere pesantemente i significati attribuiti da parte dei destinatari nel corso del loro lavoro di interpretazione.

Le cause di tale fenomeno sono molteplici: difetti relativi al medium di trasmissione, ambiguità e contraddizioni semantiche dovute alla cattiva costruzione del messaggio, imperfetta comprensione dell'enciclopedia del destinatario da parte dell'emittente, delegittimazione totale o parziale dell'emittente da parte del destinatario. La causa più importante, tuttavia, è probabilmente rappresentata dalle interferenze prodotte dall'eccessiva quantità e dalla natura entropica dei messaggi circolanti in quel denso flusso comunicativo che caratterizza l'odierno sistema dei media.

La mancanza di simmetria enciclopedica deve comunque essere ridotta al minimo nella comunicazione di un messaggio, perché comporta una notevole perdita di risorse. Si cerca generalmente di rimediarvi utilizzando la "ridondanza", cioè la ripetizione dei messaggi. Uno strumento cui la pubblicità fa spesso ricorso, anche se presenta dei rischi, come la produzione di effetti di forte banalizzazione sull'immagine di marca.

\footnotetext{
${ }^{33}$ Ibidem.

${ }^{34}$ ECO, U. (1984): Semiotica e filosofia del linguaggio, Torino, Einaudi.
} 
Applicheremo ora il modello che abbiamo presentato per interpretare i messaggi della pubblicità a quello che probabilmente è il caso più classico nell'ambito dell'analisi semiotica della pubblicità: Panzani (cfr. Figura 7). Roland Barthes ${ }^{35}$ ha infatti sviluppato per primo negli anni Sessanta la possibilità di un'analisi del linguaggio visivo e lo ha fatto proprio attraverso l'individuazione dei significati presenti nell'annuncio stampa della marca Panzani.

Fig. 7

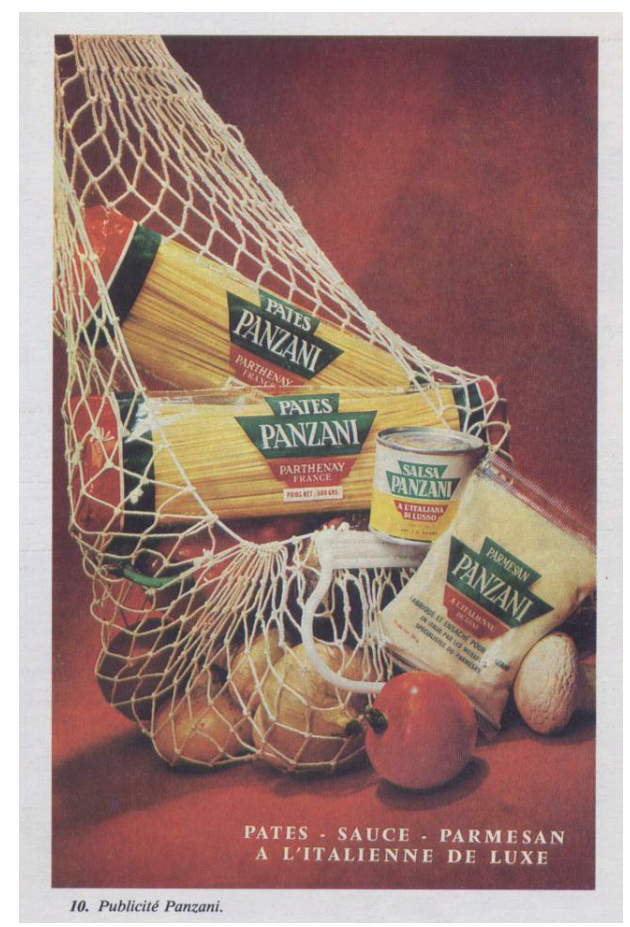

\section{Un esempio classico: Panzani}

Secondo Barthes, ogni messaggio di tipo visivo è articolato su due livelli di funzionamento (iconico codificato o denotato e iconico non codificato o connotato), i quali vanno ad aggiungersi a quel livello propriamente linguistico che caratterizza il testo verbale di qualsiasi messaggio. All'interno del livello connotativo dell'annuncio Panzani, è possibile individuare quattro significati (o segni connotati), che insieme costituiscono il messaggio culturale complessivo che va ad aggiungersi a quello linguistico (la parola «Panzani»). Si tratta di questi significati:

- il ritorno dal mercato (suggerito dalla borsa della spesa semiaperta e dalla freschezza dei prodotti in essa contenuti);

${ }^{35}$ BARTHES, R. (1964): «Retórica de la imagen », in BARTHES, R., Lo obvio y lo obtuso. Imágenes, gestos, voces, Barcelona, Paidós, 1992 (1982). 
- l'italianità (espressa dalle verdure acquistate, i cui colori richiamano quelli della bandiera italiana);

- il «servizio culinario totale» (comunicato attraverso l'idea che Panzani offre tutto ciò che serve per preparare il pasto e instaurando una equivalenza tra il prodotto naturale e quello confezionato);

- l'artisticità (grazie al richiamo alla tradizione pittorica della "natura morta").

L'analisi dell'annuncio Panzani effettuata da Barthes ha avuto sicuramente il pregio dell'originalità, in quanto ha aperto una nuova strada per l'analisi dei messaggi pubblicitari. Vista però con gli occhi contemporanei presenta anche una certa confusività, perché «unisce in un medesimo significante elementi differenti come gli oggetti e i colori $\rangle^{36}$. Soprattutto però il suo problema è che è stata superata dallo sviluppo delle conoscenze che si è avuto negli ultimi anni all'interno della semiotica. In tale ambito si è acquisita infatti la consapevolezza che è necessario mettere in luce, più che una serie di segni connotati, l'articolazione complessiva del messaggio, la quale comprende componenti profonde che non sono immediatamente percepibili.

Gianfranco Marrone ${ }^{37}$ ha mostrato a tale proposito come nell'immagine dell' annuncio Panzani sia presente una narrazione di tipo implicito: la partenza, il viaggio, l'azione (scelta dei prodotti, loro accostamento, acquisto), il viaggio di ritorno, l'allestimento della pietanza, il consumo. Si tratta di una narrazione relativa a ciò che è stato compiuto da quel Destinante (cioè il soggetto narrativo che fa partire il racconto) che sembra essere responsabile della scelta dei prodotti e dei loro accostamenti. Nella narrazione non sono presenti degli attori umani, ma questi sono comunque operativi ai livelli profondi del messaggio. Lo spettatore può cioè ricostruire mentalmente un racconto che nell'immagine pubblicitaria non è narrato esplicitamente, ma soltanto suggerito.

Sul piano plastico, l'immagine presenta una rete della spesa che offre un insieme di prodotti frutto di una selezione. Il fatto che la rete sia tesa e penda dall'angolo superiore sinistro indica che qualcuno, al momento non visibile, la sta reggendo nello stesso istante in cui il lettore la vede ed è probabilmente colui che ha effettuato tale selezione. C'è dunque una sorta di "raddoppiamento" della presenza del Destinante: è il soggetto che in precedenza ha effettuato la selezione dei prodotti, ma anche quello che ora li sta offrendo allo spettatore che vede il messaggio. Va considerato inoltre che l'immagine colloca chiaramente tale Destinante in alto e cioè in una posizione gerarchicamente superiore. Il Destinante non può che essere dunque, come ha indicato anche lo stesso Marrone, la marca Panzani.

Va considerato inoltre che, essendo una parte della rete collocata al di fuori dell'immagine, viene ad essere attivato un processo di completamento dell'immagine da parte del lettore del messaggio. Siamo dunque in presenza della figura retorica della aposiopesi (o reticenza), che sfrutta il meccanismo percettivo messo in luce dalla teoria della Gestalt per cui un messaggio incompleto tende a stimolare il suo completamento.

\footnotetext{
${ }^{36}$ JOLY, M. (1993 ): Introduction à l'analyse de l'image, Paris, Nathan, 88.

${ }^{37}$ MARRONE, G. (2001): Corpi sociali. Processi comunicativi e semiotica del testo, Tori-
} no, Einaudi. 
Il Destinante sembra stipulare un contratto con il Soggetto-consumatore, proponendogli dei valori che sono di tipo "ossimorico", che cioè fanno ricorso alla figura retorica dell'ossimoro per tenere insieme significati contrapposti. Comunicano infatti «natura confezionata», perché i prodotti realizzati dall'industria Panzani vengono percepiti come freschi grazie agli ortaggi naturali che si trovano insieme ad essi all'interno della borsa della spesa. Dunque, la loro immagine è anche il risultato di un processo di comunicazione che si sviluppa sul piano metonimico: i prodotti in scatola di Panzani sono genuini come i veri pomodori di cui sono costituiti.

Secondo Marrone, la struttura narrativa di questo annuncio stampa si regge sulla presenza nel livello più profondo dell'annuncio del valore «lusso» (esplicitato anche dal payoff «à l'italienne de luxe» che si trova in basso a destra sotto l'elenco dei prodotti Panzani: paste, salsa, parmigiano). Egli può dire ciò perché diversi elementi convergono nel comunicare dei significati di lusso, come il fondo rosso della scena (che rimanda al panno sul quale vengono abitualmente presentati i gioielli preziosi o al sipario tipico della scena teatrale) e la situazione presentata, che appare surreale e fa pensare a un sogno piacevole.

Ma Marrone non si accorge, come d'altronde era accaduto anche allo stesso Barthes, che il messaggio contiene in profondità soprattutto il significato di maternità. La rete infatti è una evidente metafora sia del ventre che del seno materno. D'altronde, l'intero mondo presentato nell'immagine è profondamente femminile. Sia sul piano simbolico, dove la rotondità abbondante della rete (che rimanda anche alla figura della cornucopia) fa pensare a una madre generosa, una mamma insomma che si presenta come un vero e proprio modello archetipico di tutte le mamme. Sia sul piano concreto e sociale, perché tradizionalmente è la donna di casa ad acquistare i prodotti e preparare i cibi. E ciò era particolarmente vero in quegli anni Sessanta nei quali l'annuncio Panzani è stato pubblicato sui giornali ed è stato analizzato da Barthes. Dunque tale annuncio si rivolge probabilmente a quella casalinga che va a fare la spesa al mercato, acquista i prodotti e in seguito offre ai membri della sua famiglia tutto ciò che è presente nella rete.

Si conferma così la validità della tesi che abbiamo sostenuto e cioè che sia la maternità quel valore profondo sul quale si regge l'intero messaggio pubblicitario di Panzani. All'epoca d'altronde l'Italia vista attraverso gli occhi della cultura francese, come ha affermato lo stesso Barthes, era soprattutto un universo orale e materno. Quell'universo, cioè, che offre nutrimento e rende pertanto possibile la vita degli esseri umani.

L'analisi che è stata appena sviluppata per il classico caso Panzani aveva lo scopo di mostrare come un messaggio pubblicitario debba essere necessariamente analizzato entrando al suo interno per individuarne i significati più profondi. Dunque, applicando il modello che è stato qui illustrato, è possibile analizzare tutti i messaggi pubblicitari. Naturalmente, tale modello non potrà essere sempre impiegato nella sua totalità. $\mathrm{A}$ seconda infatti delle specifiche caratteristiche di ciascun messaggio, dovranno essere di volta in volta impiegate solamente quelle componenti del modello che si renderanno necessarie. 


\section{Riferimenti bibliografici}

BARTHES, R. (1957): Mythologies, Paris, Seuil.

- (1961): «La imagen fotográfica», in BARTHES, R., Lo obvio y lo obtuso. Imágenes, gestos, voces, Barcelona, Paidós, 1992 (1982), 11-27.

- (1964): «Retórica de la imagen », in BARTHES, R., Lo obvio y lo obtuso. Imágenes, gestos, voces, Barcelona, Paidós, 1992 (1982), 30-47.

BEASLEY, R.; DANESI, M. (2002): Persuasive Signs: The Semiotics of Advertising, Berlin-New York, Mouton de Gruyter.

BETTETINI, G. (1984): La conversazione audiovisiva. Problemi dell'enunciazione filmica e televisiva, Milano, Bompiani.

- (1985): L'occhio in vendita. Per una logica e un'etica della comunicazione audiovisiva, Venezia, Marsilio.

- (1993): Semiotica della comunicazione d'impresa, Milano, Bompiani.

BLANCHÉ, R. (1966): Structures intellectuelles. Essai sur l'organisation systématique des concepts, Paris, Vrin.

CARO, A. (2010): Comprender la publicidad, Barcelona, Trípodos.

CASETTI, F. (1986): Dentro lo sguardo. Il film e il suo spettatore, Milano, Bompiani.

CODELUPPI, V. (1989): Consumo e comunicazione. Merci, messaggi e pubblicità nelle società contemporanee, Milano, Franco Angeli.

- (ed.) (1995): La sfida della pubblicità, Milano, Franco Angeli.

- (1997): La pubblicità. Guida alla lettura dei messaggi, Milano, Franco Angeli.

- (2001a): Che cos'è la pubblicità, Roma, Carocci.

- (2001b): Il potere della marca, Torino, Bollati Boringhieri.

- (2005), Manuale di Sociologia dei consumi, Roma, Carocci.

CORNU, G. (1991): Sémiologie de l'image dans la publicité, Paris, Les éditions d'organisation.

ECO, U. (1975): La estructura ausente. Introducción a la semiótica, Barcelona, Lumen (1968).

- (1979): Lector in fabula, Milano, Bompiani.

- (1984): Semiotica e filosofia del linguaggio, Torino, Einaudi.

EUGENI, R. (1999): Analisi semiotica dell'immagine. Pittura, illustrazione, fotografia, II ed., Milano, ISU-Università Cattolica.

FLOCH, J.-M. (1993), Semiótica, marketing y comunicación. Bajo los signos, las estrategias, Barcelona, Paidós (1991).

GRANDI, R. (1987): Come parla la pubblicità. Modelli comunicativi degli spot pubblicitari, Milano, Edizioni del Sole 24 Ore.

GREIMAS, A.J. (1966): Sémantique structurale, Paris, Larousse.

- (1970): Du Sens, Paris, Seuil.

- (1983): Du Sens II. Essais sémiotiques, Paris, Seuil.

JAKOBSON, R. (1976), Ensayos de lingüistica general, Barcelona, Seix Barral (1963). JOLY, M. (1993): Introduction à l'analyse de l'image, Paris, Nathan.

LANDOWSKI, E. (2006): «Les Interactions risquées», Nouveaux Actes Sémiotiques, 101-103.

MAGARIÑOS DE MORENTIN, J.A. (1984): El mensaje publicitario, Buenos Aires, 
Hachette.

MAC RURY, I. (2009): Advertising, London-New York, Routledge.

MARRONE, G. (2001): Corpi sociali. Processi comunicativi e semiotica del testo, Torino, Einaudi.

- (2007): Il discorso di marca. Modelli semiotici per il branding, Roma-Bari, Laterza. PÉNINOU, G. (1976): Semiótica de la publicidad, Barcelona, Gustavo Gili (1972).

PIGNOTTI, L.; MUCCI E. (1978): Marchio \& Femmina. La donna inventata dalla pubblicità, Firenze, Vallecchi.

PROPP, V.J. (1974): Morfología del cuento fiaba, Madrid, Fundamentos (1928).

REMAURY, B. (2004): Marques et récits. La marque face à l'imaginaire culturel contemporain, Paris, Institut Français de la Mode-Regard.

SHANNON, C.E.; WEAVER, W. (1949): The Mathematical Theory of Communication, Urbana, University of Illinois Press.

TRAINI, S. (2008): Semiotica della comunicazione pubblicitaria, Milano, Bompiani.

VESTERGAARD, T.; SCHRØDER, K. (1985): The Language of Advertising, Oxford, Basil Blackwell.

VOLLI, U. (2003): Semiotica della pubblicità, Roma-Bari, Laterza. 